\title{
Optimization of thick-walled spherical shells at thermal and power influences
}

\author{
Stepan Litvinov ${ }^{1 *}$, Alex Beskopylny ${ }^{1}$, Lyubov Trush $^{1}$, and Serdar Yazyev ${ }^{1}$ \\ ${ }^{1}$ Don State Technical University, Rostov-on-Don, Russia
}

\begin{abstract}
The problem of optimization for thick-walled shell, experiencing temperature and power feedback. Under the influence of temperature field the properties of material of an object can change. That allows to manage the deflected mode of such objects while achieving a certain law of radial change of physical and mechanical parameters. A centrally symmetric problem of elasticity theory is studied. As a result we received a law of variation of Young's modulus, in which a spherical dome is equally stressed according to the simplified theory of Mohr. The problem was reduced to a Bernoulli differential equation. This equation was solved numerically using Runge-Kutta method of fourth order.
\end{abstract}

\section{Introduction}

Despite development of numerical methods, such as a finite-element method, analytical methods of task solutions and calculations on their basis are still relevant.

One of the most widespread classes of tasks are central symmetric $[1 ; 2]$ and the axisymmetric tasks [3-5] differing generally in some coefficients while general structure of the resolving equations is preserved.

There are numerous researches on determination of the deflected mode of cylindrical objects (pipes, reservoirs) under the influence of external pressure and temperature [6]. At that, the change of physical and mechanical parameters of a material under the influence of temperature isn't always taken into account.

The above-mentioned property can be used for solving the inverse mechanical problems: determining such a law of the modulus of elasticity change of material of a structure and its elements that would make it equal in tension and strength [7-9].

\section{Problem definition}

We consider a thick-walled shell with inner radius $a$ and outer radius $b$, experiencing the inner pressure $p_{a}$ and outer pressure $p_{b}$ (Fig. 1). The temperature of a shell varies from $T_{a}$ on its inner surface, up to 0 degrees centigrade on the outer one.

In such a case the law of temperature change will be the following:

*Stepan Litvinov: litvstep@gmail.com 


$$
T(\mathrm{r})=T_{a} \frac{a}{a-b}\left(1-\frac{b}{r}\right)
$$

It is known that stress distribution in a homogeneous construction will be uneven. Exceeding of pressure loading capacity of a homogeneous shell takes place on a small area near the inner or outer surface (depending on the correlation between temperature and pressure values). So, the structural strength of a material is underused. Creating an artificial heterogeneity will help to make a permanent stress at every point of the construction. To do so, we should reduce the modulus of elasticity of a material at the points experiencing more stress, and raise it at the points with less stress. Polymer materials provide extensive possibilities for variating the modulus of elasticity, because in such materials the modulus depends on the filler content [10]. Seeking for the law of modulus of elasticity change, in which a stressed condition is a defined one, underlies inverse construction optimization method [11].

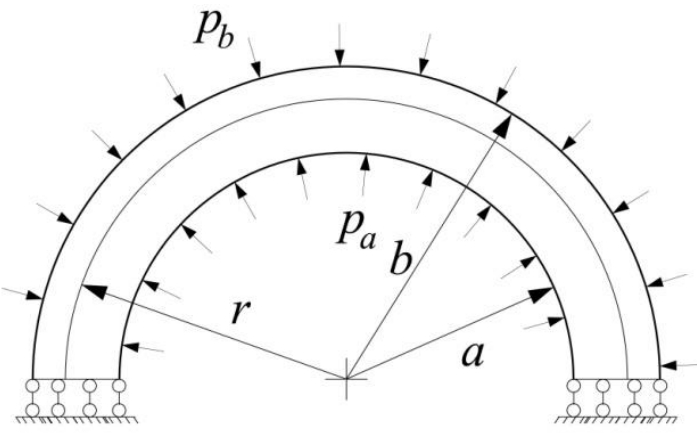

Fig. 1.A spherical shell under inner and outer pressure.

Being under the inner pressure, a spherical shell is under the centrally symmetric problem. In case of changing the modulus of elasticity along the radius, the problem is reduced to the following differential equation in terms of radial stresses [11]:

$$
\sigma_{r}^{\prime \prime}+\left(\frac{4}{r}-\frac{E^{\prime}}{E}\right) \cdot \sigma_{r}^{\prime}-\frac{1}{r} \cdot \frac{2(1-2 v)}{1-v} \cdot \frac{E^{\prime}}{E} \sigma_{r}=-\frac{2 E \alpha_{T} T^{\prime}}{r(1-v)},
$$

where $\alpha_{T}$ is a linear thermal expansion coefficient.

The prime mark here denotes the derivative with respect to $r$ coordinate. As a strength criterion we shall use a simplified Mohr theory, which is suitable for materials, having different tensile and compression reactions. Equivalent (estimated) tension for axisymmetric problem can be written down as following:

$$
\sigma_{\text {equiv }}=\sigma_{\theta}-k \sigma_{r}
$$

where $k=R_{p} / R_{c}$ is a ratio of material tension strength $R_{p}$ to material compression strength $R_{c}$.

The equivalent tension for equally stressed shell must be constant across the entire thickness of the construction:

$$
\frac{d \sigma_{\text {equiv }}}{d r}=\frac{d \sigma_{\theta}}{d r}-k \frac{d \sigma_{r}}{d r}=0
$$

The interconnection of radial and circumferential tensions for a spherical shell is as follows [11]: 


$$
\sigma_{\theta}=\frac{1}{2} r \sigma_{r}^{\prime}+\sigma_{r}
$$

Let's substitute (5) in (4):

$$
r \sigma_{r}^{\prime \prime}=(2 k-3) \sigma_{r}^{\prime}
$$

The general solution of the equation (6) when $k \neq 1$ is as follows:

$$
\sigma_{r}=\frac{C_{1} r^{2 k-2}}{2 k-2}+C_{2},
$$

where $C_{1}$ and $C_{2}$ are arbitrary constants, defined on the basis of the boundary conditions:

$$
\begin{gathered}
\sigma_{r}(a)=-p_{a}, \quad \sigma_{r}(b)=-p_{b} \\
C_{1}=\frac{(2 k-2)\left(p_{b}-p_{a}\right)}{a^{2 k-2}-b^{2 k-2}}, C_{2}=\frac{p_{b} a^{2 k-2}-p_{a} b^{2 k-2}}{b^{2 k-2}-a^{2 k-2}}
\end{gathered}
$$

When we substitute (7) in (2), we will get the following differential equation in term of modulus of elasticity:

$$
E^{\prime}\left(B r^{2 k-2}+C\right)=E^{2} \frac{2 \alpha}{1-v} T^{\prime}+E A r^{2 k-3}
$$

where $A=C_{1}(2 k+1), B=C_{1}\left(1+\frac{1-2 v}{(1-v)(k-1)}\right), C=C_{2} \frac{2(1-2 v)}{1-v}$

The equation (9) is the Bernoulli differential equation. This equation is: $E^{\prime}=f(r, E)$. So, it is possible to solve it numerically using Runge-Kutta fourth-order method [12-15].

The first order differential equation needs one boundary condition. The modulus of elasticity on the inner surface $E_{0}=E(a)$ may be arbitrary. It is convenient to set it to one. Then we will get sets of curves $E(r) / E_{0}$.

\section{Results and Discussion}

The test problem was solved when $a=15 \mathrm{~cm}, \quad b=25 \mathrm{~cm}, \alpha_{T}=10^{-5} 1 / \mathrm{deg}, \quad v=0,3$, $k=0,5$, inner pressure $p_{a}=10 \mathrm{MPa}$, outer pressure $p_{b}=0, T_{a}=80{ }^{0} \mathrm{C}$.

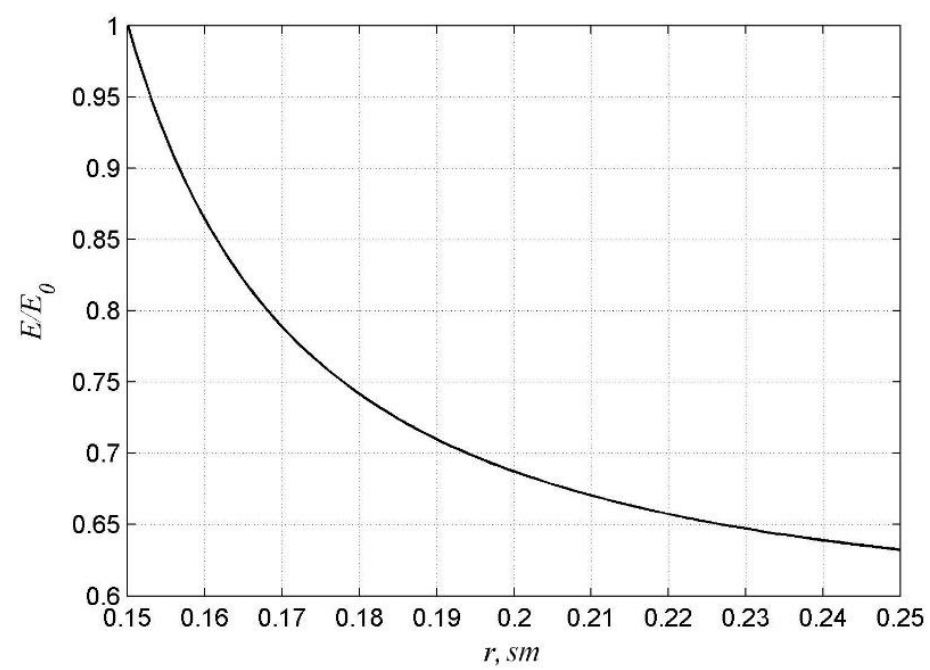

Fig.2.Change of Young's modulus of an equally-stressed shell. 


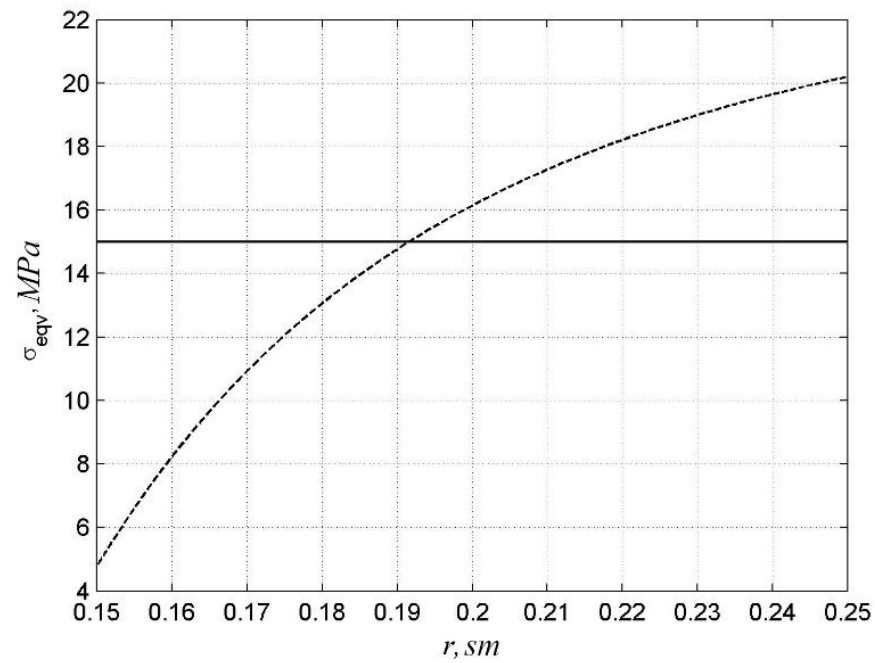

Fig.3. Distribution of equivalent tension in the thickness of homogeneous (dashed line) and heterogeneous (solid line) structures.

Fig. 2 displays the Change of Young's modulus graph $E(r) / E_{0}$ of an equally-stressed shell. Fig. 3 illustrates the change of equivalent tension in the thickness of homogeneous (dashed line) and heterogeneous (solid line) structures. The graphs show that a maximum design stress dropped from, 20,2 $\mathrm{MPa}$ to $15 \mathrm{MPa}$. So, the pressure loading capacity of a shell with ordered thickness has risen to $35 \%$.

\section{Conclusion}

The use of material in homogeneity may well benefit the durability and wear resistance property of structures and their elements. In this case the object's potential is involved as much as possible.

Difficulties can arise when getting the set dependences of change of the radial module of elasticity. There are several methods for achieving the purpose in view: 1. operating with distribution of particles on object radius due to centrifugal action of a cylinder; 2 . due to the temperature field influence leading to change of physical and mechanical properties of material of an object.

\section{References}

1. V.I. Andreev, D.A. Kapliy, Procedia Engineering, 111, 36-41(2015)

2. B.M. Yazyev, A.S. Chepurnenko, A.V. Muhanov, Inzhenernyj vestnik Dona, 3, 26, 141 (2013)

3. V.I. Andreev, Matec web of conferences, 05008 (2016)

4. V.I. Andreev, Procedia Engineering, 153, 32-36 (2016)

5. V.I. Andreev, L.S. Polyakova, Advanced Materials and Structural Eng. Proceedings of the Int. Conf. on Advanced Materials and Eng. Structural Technology, 715-718 (2015)

6. A.S. Chepurnenko, A.N. Beskopylnyi, B.M. Jazyev, V.I. Andreev, MATEC, 86, 06059 (2016) 
7. A.E. Dudnik, N.I. Nikora, A.S. Chepurnenko, Nauchnoe obozrenie, 11,74-78 (2015)

8. V.I. Andreev, A.S. Chepurnenko, B.M. Yazyev, Advanced Materials Research, 869872 (2014)

9. A.E. Dudnik, A.S. Chepurnenko, N.I. Nikora, A.S. Denego, Inzhenernyj vestnik Dona, 2, $136(2015)$

10. B.M. Yazyev, Nonlinear creep of continuously inhomogeneous cylinders (1990)

11. V.I. Andreev, Some problems and methods of mechanics of heterogeneous solids: monograph (Publishing house ASV, 2002)

12. A.S. Chepurenko, V.I. Andreev, B.M. Yazyev, Vestnik MGSU, Proceedings of Moscow State University of Civil Engineering, 56-61 (2013)

13. A.S. Chepurnenko, B.M. Yazyev, Nauchnoe obozrenie, 202-204 (2012)

14. A.E. Dudnik, A.S. Chepurnenko, N.I. Nikora, A.S. Denego, Inzhenernyj vestnik Dona (2015)

15. V.I. Andreev, A.S. Chepurnenko, B.M. Yazyev, Trans Tech Publications, Switzerland, $257-260$ (2014) 